\title{
CONSTRAINTS TO THE MASS OF A BLACK HOLE AT THE GALACTIC CENTRE
}

\author{
L.M. Ozernoy \\ Lebedev Physical Institute, Moscow, USSR
}

Many speculations related to a hypothesis that there is a very massive black hole at the centre of our Galaxy have appeared during the last years. This hypothesis is based, firstly, on the opinion that the formation of a massive black hole as a result of dynamical evolution of galactic nuclei is inevitable and, secondly, on the attempts to explain the nature of the "point source" at the Galactic centre (see Oort, 1977 and references therein). However both these approaches could not predict with any confidence the black hole mass. The estimates range from $10^{7}-10^{11} M_{0}$ (Novikov and Thorne, 1973) to $10^{4} \mathrm{M}_{0}$ (Shklovskii, 1976).

I should like to propose a method to obtain an upper limit to the black hole mass based on the calculations of the inevitable growth of the mass in the course of tidal disruption of stars surrounding the assumed hole at the Galactic centre. The calculations of secular growth of the black hole formed presumably $10^{10}$ yrs ago in the Galactic nucleus were made recently by Dokuchaev and Ozernoy (1977a) under the following assumptions : (i) stars in the nucleus have a Maxwellian velocity distribution, and the rotation of the nucleus is negligibly small; (ii) most part of the gas from disrupted stars is accreted eventually onto the hole; ( $i$ ii) the main parameters of the Galactic core (its radius $R_{c} \simeq 1 \mathrm{pc}$ and star density $n_{c} \simeq 10^{7} \mathrm{pc}^{-3}$ ) do not change appreciably during secular evolution of the black hole.

The character of the growth of the black hole mass is shown in Fig. 1. As long as the black mass is comparatively small, its tidal forces disrupt neighbouring stars with a rate $N \simeq 6 \times 10^{-7}\left(\mathrm{M}_{\mathrm{h}} / 10^{3} \mathrm{M}_{0}\right)^{4 / 3}$ $\mathrm{yr}^{-1}$ (Hi11s, 1975; Dokuchaev and Ozernoy, 1977b). After 10-100 stars are disrupted, their remnants in form of gaseous discs which will be inclined to each other under different angles, will provide an effective accretion of the gas onto the hole. Afterwards accretion which will be apparently quasi steady rather than intermittent, will proceed at an 


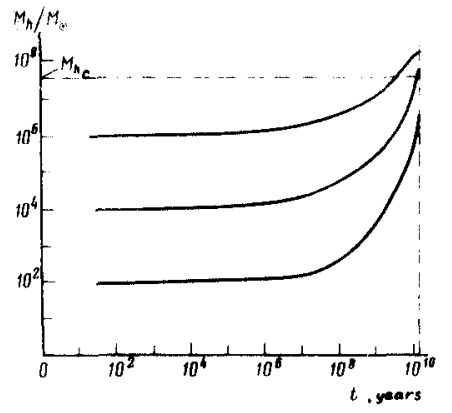

now at a lower rate $\hat{N} \simeq 4 \times 10^{-3} \mathrm{yr}^{-1}$. amount determined by the rate of star disruption. When the mass $M_{2} \simeq 3 \times 10^{7} \mathrm{M}$ is reached, disruption of stars due to collisions begins to prevail over tidal disruption. And, finally, when the black hole mass will become comparable with mass of the core (i.e. when the concentration of stars diminishes noticeab1y), tidal disruption will again become dominant over star collisions, but will proceed

As it is seen from Fig. 1, an appreciable growth of the black hole mass during $10^{10} \mathrm{yr}$ is possible only in the case if the initial mass was greater than ca. $10^{2} \mathrm{M}$. An important additional feature is shown in Fig. 2 which gives the relation between the initial black hole mass $M_{h}(0)$ and its expected present value $M_{h}\left(10^{10}\right.$ yr) caused by accretion.

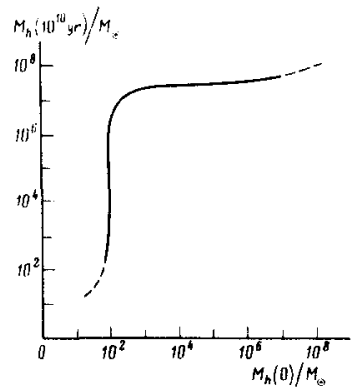
As one can see, the mass increases during $10^{10}$ yr to a value $\mathrm{M}_{\mathrm{h}} \sim\left(4 \times 10^{6}-10^{8}\right) \mathrm{M}_{\text {e wich de- }}$ pends weakly on the initial mass provided that the latter exceeds only slightly $10^{2} \mathrm{M}$.

Calculations of the ${ }_{2}$ black hole luminosity $\mathrm{L}=\varepsilon \mathrm{m}_{\star} \mathrm{c} N$ (where $\varepsilon \sim 0.1$ is the efficiency of mass-to-energy production during accretion of a disrupted star of the mass $m_{*}$ ) indicate that this luminosity does not exceed the total non-stellar radiation of the Galactic nucleus $\mathrm{L} \leqslant 10^{42} \mathrm{ergs} / \mathrm{s}$ only if the black hole mass is not larger than $\mathrm{M}_{\mathrm{h}} \sim 10^{4} \mathrm{M}_{0}$ (Ozernoy, 1976). Combining this with the above mentioned results concerning the secular growth of the black hole mass, it is possible to reduce this upper limit to a much lower value $M_{h} \leqslant 10^{2} M_{\odot}$. Indeed, as it is seen $f_{r} 8^{m} F i g .2$, even the minimal value of the final black hole mass $M_{h}\left(10^{10} \mathrm{yr}\right) \simeq 4 \times 10^{6}$ $M_{\text {f }}$ exceeds appreciably the acceptable upper limit $\mathrm{M}_{h} \sim 10^{4} \mathrm{M}_{\odot}$ provided that the initial mass $M_{h}(0)>10^{2} M_{0}$. Of course, there is a possibility that a black hole in the Galactic centre was formed so recently that its present mass lies within the interval of $\left(10^{2}-10^{4}\right) \mathrm{M}$. However, this possibility seems to be rather artificial.

Let us discuss briefly other assumptions and simplifications 1 isted 
above which could change the upper limit obtained.

(i) Re-population of the "loss-cone" by stars due to diffusion of their orbits could depend on the rotation of the core only at a large anisotropy of star velocities. As for the situation in the nucleus of M31 (which dynamical parameters are very similar to our own) its rotational velocity is much smaller than the velocities of chaotic motions of stars (e.g. Ruiz, 1976). This makes it quite reasonable to neglect the rotation, in a first approximation, in estimates of the tidal disruption rate.

(ii) The possibility of partial ejection of gas from disrupted stars out of the sphere of its spreading as a result, e.g., of thermal flares induced by the tidal forces (Lidskii and Ozernoy, 1977) was not taken into account. On the other hand, the interaction of stars with elongated gas clouds (remnants of disrupted stars) was neglected a1so. Their collisions lead to a decrease of orbital angular momentum of the stars and, consequently, to a more rapid filling of the loss-cone. These processes work in opposite directions and compensate partially each other.

(iii) Although the assumption that the main dynamical parameters of the nuclear core are constant during its life is an oversimplification, a detailed analysis indicates (Dokuchaev and Ozernoy, 1977a) that it is not too bad.

A stringent upper limit $\mathrm{M}_{\mathrm{h}} \leqslant 10^{2} \mathrm{M}_{\theta}$ to the black hole mass in the Galactic centre raises an interesting question : why did the dynamical evolution of the nucleus lead to a small, if any, mass of the black hole? A possible answer, according to results of Dokuchaev and Ozernoy (1977c), may lie in the formation of a large number of close binary systems which may be the main factor preventing both collapse of the core and the formation of a massive black hole there.

Regardless of the eventual explanation of its low value, the upper limit to the black hole mass in the Galactic nucleus appears to be in contradiction 1) with the hypothesis that nuclei of normal galaxies are dead quasars (Lynden-Be11, 1969) and 2) with the hypothesis that relic black holes may be the main factor of galaxy formation, since the mass of a relic black hole must have grown to $\sim 10^{7} \mathrm{M}$ by the present time if it served as a centre for the formation of the Galaxy (Ryan, 1972).

To summarize, I conclude that the stringent upper limit to the mass of a black hole in the Galactic centre mentioned above is of importance for the dynamical history of the Galaxy. 


\section{REFERENCES}

Dokuchaev, V.I. and Ozernoy, L.M. 1977a, Preprint Lebedev Physical Institute No. 137; Pis'ma Astr. Zh. 3, 391

Dokuchaev, V.I. and Ozernoy, L.M. 1977b, Preprint Lebedev Physica1. Institute No. 133; Zh. Exp. Teor. Fiz. (in press)

Dokuchaev, V.I. and Ozernoy, L.M. 1977c, Preprint Lebedev Physical Institute No. 134 ; Astr. Zh. (in press)

Hi11s, J.G. 1975, Nature 254, 295

Lidskii, V.V. and Ozernoy, L.M. 1977, Pis'ma Astr. Zh. 3, No. 12

Lynden-Be11, D. 1969, Nature 223, 690

Novikov, I.D. and Thorne, K.S. 1973, In "Black Holes", Eds C. de Witt and B. de Witt (Gordon \& Breach, N.Y.), p. 343

Oort, J.H. 1977, Ann. Rev. Astr. Astrophys. 15 (in press)

Ozernoy, L.M. 1976, Observatory 96, 67

Ruiz, M.T. 1976, Astrophys. J. $2 \overline{07}, 382$

Ryan, M.P. 1972, Astrophys. J. $\underline{177}$, L79

Shklovskii, I.S. 1976, Pis'ma Astr. Zh. 2, No. 7, p. 3 